\title{
The VLA Sky Survey
}

\author{
Eric J. Murphy*, and the VLASS Survey Science Group \\ Infrared Processing and Analysis Center, Caltech, MC 314-6, Pasadena, CA 91125, USA \\ National Radio Astronomy Observatory, 520 Edgemont Road, Charlottesville, VA 22903, USA \\ E-mail: emurphyanrao.edu
}

The VLA All-Sky Survey (VLASS) builds upon the newly upgraded Karl G. Jansky Very Large Array (VLA) to employ its enhanced bandwidth, time resolution, and survey speed to carry out a next-generation sky survey. VLASS will map the sky at $\delta>-40^{\circ}$, covering a total of 33,885 square degrees, with 2". 5 angular resolution using the VLA B-array at 2-4 GHz, with an expected rms of $69 \mu \mathrm{Jy} / \mathrm{beam}$ in the final images. The survey will be carried out over three epochs spanning 32 months, providing a synoptic view of the dynamic radio sky similar to those now available through the new generation of synoptic imagers at other wavelengths. Consequently, VLASS will provide unique measurements of the radio sky at key epochs and sensitivity levels between that from FIRST and NVSS and the new upcoming radio surveys. This will be a critical enabler for early identification and filtering for the most interesting transient events. In this proceeding we highlight some of the key science that will be delivered by VLASS that make optimal utilization of the Jansky VLA's unique capabilities: high resolution imaging and exquisite point-source sensitivity, critical for source identification; wide bandwidth coverage, enabling instantaneous spectral index determination; and full polarimetry with good performance even in lines of sight with high Faraday depth, enabling instantaneous rotation measure and Faraday structure determinations.

EXTRA-RADSUR2015 (*)

20-23 October 2015

Bologna, Italy

(*) This conference has been organized with the support of the Ministry of Foreign Affairs and International Cooperation, Directorate General for the Country Promotion (Bilateral Grant Agreement ZA14GR02 - Mapping the Universe on the Pathway to SKA)

\footnotetext{
*Speaker.
} 


\section{Background}

The recent upgrade of the Karl G. Jansky Very Large Array (VLA) has effectively transformed it into a new telescope, with an order of magnitude improvement in sensitivity, spectral coverage, and versatility (Perley et al., 2011). Consequently, the capabilities required for the VLA Sky Survey $\left(\right.$ VLASS $^{1}$ ) have largely been proven in past and ongoing science programs, making this the ideal time to utilize the VLA for a science survey that will both inform future Jansky VLA PI science for decades to come, while simultaneously planting radio astronomy firmly within the multi-wavelength framework of astronomical research. VLASS is a community-driven project that will use this powerful new platform to conduct a next-generation radio sky survey. By leveraging the enhanced time domain, polarization, and spectral capabilities of the new VLA, VLASS will vastly expand on the pioneering sky surveys [FIRST, Becker et al. (1995); NVSS, Condon et al. (1998)] produced by the original VLA. VLASS was developed through unprecedented community involvement starting in September 2013, including public workshops, white papers, and debate within working groups of more than 200 multi-wavelength astronomers. The resulting survey design will deliver high impact scientific return in targeted science themes, while producing a new gold standard as a radio reference image for astronomers of all kinds. VLASS will engage radio astronomy experts, multi-wavelength astronomers, and citizen scientists alike, leaving a lasting legacy.

To summarize, VLASS is an "All-Sky" survey $\left(\delta>-40^{\circ}\right)$ that will cover 33,885 square degrees. It will be observed with 2". 5 angular resolution using the VLA B-array at $2-4 \mathrm{GHz}$, with an expected rms of $69 \mu \mathrm{Jy} /$ beam in the final images. The survey will require $\sim 5,400$ hours of observation time, which will be distributed over three epochs spanning 32 months. Data from VLASS will be available in the NRAO archive immediately with no proprietary period and science data products will be provided to the community in a timely manner. The design of VLASS paid extremely close attention to future Square Kilometer Array (SKA) pathfinders, leading to a survey that will both stand out, and be complementary to those other pathfinder surveys at $1.4 \mathrm{GHz}$. Below we present some key science highlights from VLASS.

\section{Key Science Goals}

\subsection{Imaging Galaxies Through Time and Space}

Active Galactic Nuclei (AGN), particularly in radio-mode, are thought to be responsible for switching off star-formation in massive galaxies, but our understanding of AGN and their influence on galaxy evolution is far from complete. The optimal combination of VLASS sensitivity and spatial resolution will provide a statistically significant population of radio galaxies and AGN with information on morphology, polarization, and spectral indices, revealing the relationship between nuclear activity and its environment across a broad range of redshift and luminosity. Radio-detected AGN populations will expand by a factor of 10 with VLASS.

\footnotetext{
${ }^{1} \mathrm{https}: / /$ science.nrao.edu/science/surveys/vlass
} 


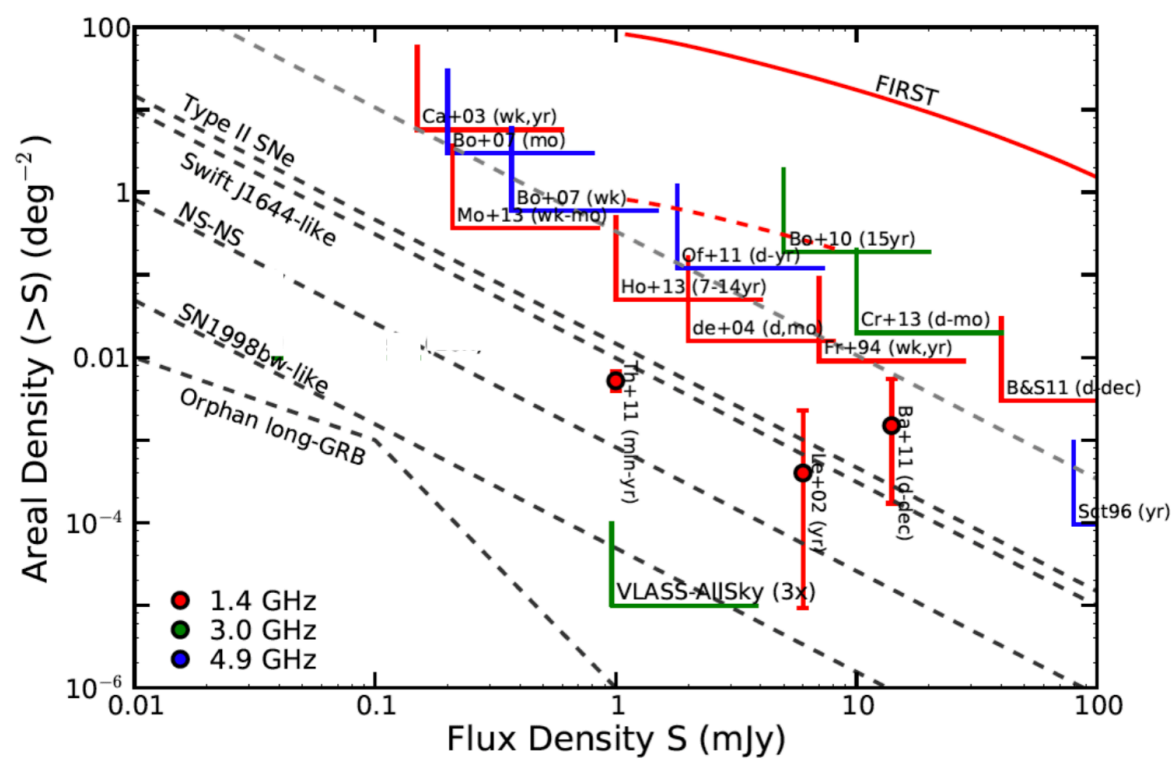

Figure 1: The phase space of explosive extragalactic radio transients. The dashed black lines give the instantaneous source counts for different classes of transients at $3 \mathrm{GHz}$. Wedges indicate upper limits to the transient rates from previous surveys, and errorbars $(2 \sigma)$ are transient rates for past detections. The markers are color-coded according to observing frequency. The limits shown for VLASS represent the expected cumulative number of detected transients, with the requirement that a transient event must be detected at $>10 \sigma$ in a single epoch, to overcome thermal noise fluctuations in the data when comparing 2 epochs ( $>10^{11}$ synthesized beams per epoch) as well as image artifacts that masquerade as transients. Even with a conservative $10 \sigma$ cut, VLASS will increase the detection yield for extragalactic transients by an order of magnitude.

\subsection{Hidden Explosions}

VLASS will be the first synoptic radio survey to systematically characterize the dynamic sky at radio frequencies to a meaningful depth, targeting both Galactic and extragalactic transient populations. A key goal is the unbiased determination of the rate of explosive events in the local universe (see Figure 1), overcoming the biases associated with the dust obscuration of supernovae at optical wavelengths and the highly collimated nature of the gamma-ray emission associated with both long and short gamma-ray bursts. The latter is particularly exciting with only one tentative detection of an off-axis ("orphan") afterglow of a gamma-ray burst at any wavelength (Tanvir et al., 2013). Extrapolating from recent calculations (Metzger et al., 2015), VLASS is predicted to detect $\sim 30$ off-axis long gamma-ray bursts and $\sim 20$ off-axis short gamma-ray bursts, the latter simultaneously being electromagnetic counterparts to the gravitational wave population that are now starting to be uncovered by Advanced LIGO.

\subsection{Faraday Tomogrpahy of the Magnetic Sky}

VLASS will open completely new regions of parameter space for the study of the magnetoionic medium in our own Milky Way, in AGNs, galaxies, and galaxy clusters across a wide range 


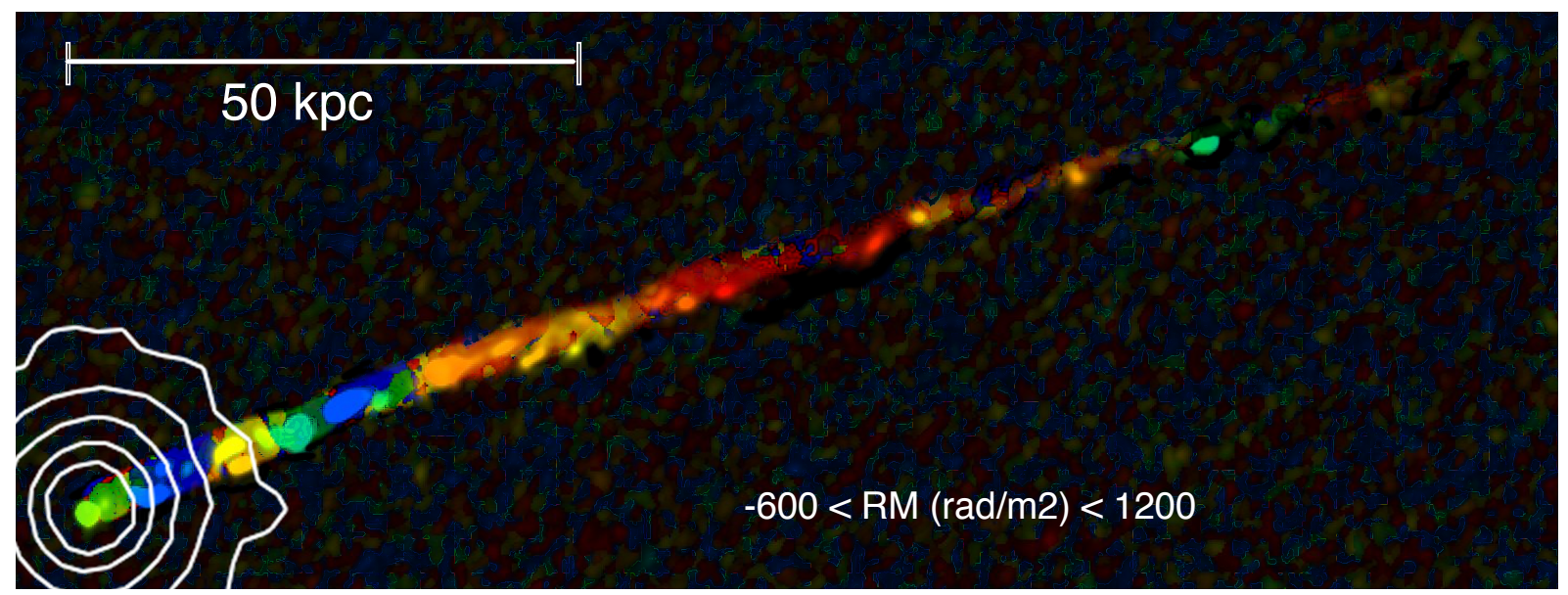

Figure 2: Polarized intensity 2-4 GHz image, color-coded by RM, for the $\sim 700 \mathrm{kpc}$ jet in Abell 2256 (L. Rudnick, F. Owen, J. Eilek, in prep;; Owen et al., 2014). Most RM patches are depolarized and not visible at $1.4 \mathrm{GHz}$. Enormous jumps on $\sim 10 \mathrm{kpc}$ scales are best explained as entrained thermal material, although this appears inconsistent with the very tight jet collimation. Many currently unpolarized sources at $1.4 \mathrm{GHz}$ will likely show such high RMstructures in the VLASS.

of redshifts (e.g., see Figure 2). It will provide the first large samples of polarization and Faraday rotation maps and complex Faraday structure (2-3 orders of magnitude more sources than currently available). These will enable the first systematic investigation of the interactions between thermal and relativistic plasmas in radio sources in different environments. Previously undetected populations of sources are also expected to be revealed with extremely large Faraday depths and strong depolarizations.

\subsection{Peering Through our Dusty Galaxy}

VLASS will complement and extend surveys of our Galaxy through dust-obscured and absorbed environments. Both thermal and non-thermal sources will be addressed, including: searches for exotic radio pulsars, overcoming known selection biases from traditional search techniques; studies of coronal magnetic activity on cool stars, probing dynamo processes and particle environments around those stars; and "book-end" studies of stars at the beginning and end of their lives, through searches for HII regions and planetary nebulae, respectively. The total size of these populations is expected to increase by $10 \times$, from $\sim 10^{4}$ to $\sim 10^{5}$.

\subsection{An All-Sky Radio Atlas}

The availability of high-resolution, high-sensitivity maps and catalogs of the entire radio sky will have broad impact on the astronomy community at all wavelengths, especially given the traditional difficulty of working with interferometric synthesis data. The previous VLA sky surveys, NVSS and FIRST, are by far the most highly cited VLA projects, with more than 4000 combined citations. Of the papers that cite FIRST or NVSS, 90\% use the archival data products. Furthermore, the data from these 20-year-old surveys continues to be heavily used: the FIRST image cutout server delivers the equivalent of a 3-minute VLA observation every 12 seconds, and every 
10 days it distributes snapshots with a total exposure time equivalent to the entire observing time invested in FIRST.

Table 1: VLASS Basic Data Products (to be provided by NRAO)

\begin{tabular}{l|l|l|l}
\hline Type & Description & Size & Notes \\
\hline \hline Visibilities & Immediate & $489 \mathrm{~TB}$ & Raw data and calibration products. \\
\hline \multirow{3}{*}{ Images } & Quick-Look & $29 \mathrm{~TB}$ & Stokes I and uncertainty. \\
& $\begin{array}{l}\text { Single-epoch } \\
\text { Cumulative }\end{array}$ & $\begin{array}{l}101 \mathrm{~TB} \\
48 \mathrm{~TB}\end{array}$ & $\begin{array}{l}7 \text { images covering Stokes I,Q,U, } \alpha_{\mathrm{I}} \text {, and uncertainties. } \\
\text { As above + curv I, tapered images (1/3 nominal resolution). }\end{array}$ \\
\hline \multirow{3}{*}{ Image Cubes } & $\begin{array}{l}\text { Coarse } \\
\text { single-epoch } \\
\text { Coarse } \\
\text { cumulative }\end{array}$ & $101 \mathrm{~TB}$ & $\begin{array}{l}\text { Source cutouts: 5 images (I,Q,U and uncertainties) for 14 } \\
128 \mathrm{MHz} \text { bands. Only 10\% of sky ( } \sim 10^{6} \text { sources). } \\
\text { As for single-epoch coarse cubes, plus a tapered set of images. }\end{array}$ \\
& $\begin{array}{l}\text { Fine } \\
\text { cumulative }\end{array}$ & $119 \mathrm{~TB}$ & $\begin{array}{l}\text { As for cumulative coarse cubes, but for } 179 \times 10 \mathrm{MHz} \text { chan } \\
\text { nels and cut outs for 2.5\% of whole sky. }\end{array}$ \\
\hline \multirow{2}{*}{ Catalogs } & $\begin{array}{l}\text { Single-epoch } \\
\text { Cumulative }\end{array}$ & $\begin{array}{l}\text { Small } \\
\text { Small }\end{array}$ & $\begin{array}{l}\text { Location, shape, brightness, spectral index, and polarization. } \\
\text { As for single-epoch case. }\end{array}$ \\
\hline
\end{tabular}

\section{Data Products}

Details of the data products, observations, and implementation plan for carrying out and processing the survey are given in the VLASS Technical Implementation Plan (TIP ${ }^{2}$ ) document. Here we briefly describe the anticipated data products to be generated for VLASS. In summary, the basic data products (BDPs) from VLASS include visibilities, images, and spectral cubes (Table 1). In addition to these BDPs, there is an ongoing community effort to enhance these products with a suite of Science Ready Data Products by developing algorithms and pipelines, processing survey data, and archiving and delivering the SRDPs to the community in a sustainable manner for years to come. A tentative list of such SRDPs that will greatly enhance the VLASS legacy include:

- Enhanced catalogs containing: Associations and classifications of detections, polarimetric information for multiple components and assembled sources, a robust/rapid transient identifications with quick-look data.

- Transients: Rapid announcements with initial classifications, along with time cut-outs of visibility data for sub-second transients.

- Polarimitry: Faraday cube cut-outs of complex spectrum with $\delta \mathrm{RM}=10 \mathrm{rad} \mathrm{m}^{-2}$.

- Archiving: Single-epoch images, cumulative images, and spectral cubes; data services to allow for querying, band-merging, and associating.

\footnotetext{
${ }^{2}$ https://www.authorea.com/users/4076/articles/8161/_show_article
} 


\section{Summary}

VLASS finds its place within this tried and true tradition of modern astronomy. Analysis of the statistics from NVSS and FIRST (as with the Hubble Deep Fields) clearly indicate that the impact on PI science from radio community surveys is as positive as those from Hubble. This is true not only in terms of the extensive usage of these data by wide sections of the community, but also due to the new inquiry driven PI science they enable, that could not otherwise have been conceived or survived the proposal process without the critical enabling data and demonstration science from the surveys. For instance, VLASS will provide an important and unique reference atlas for the science that will be enabled by a new suite of multi-wavelength surveys and observatories coming online now through the end of the decade across the electromagnetic spectrum and beyond - Pan-STARRS, ALMA, DES, JWST, eROSITA, Advanced LIGO/VIRGO, LSST, to name but a few. Lastly, while unquestionably delivering forefront science, VLASS will also accelerate the integration of radio astronomical data into the multi-wavelength astronomical community, greatly increasing the number of astronomers who will be in an optimum position to make substantial use of the SKA when it comes online.

\section{Acknowledgments}

EJM acknowledges support of the Ministry of Foreign Affairs and International Cooperation, Directorate General for the Country Promotion (Bilateral Grant Agreement ZA14GR02 - Mapping the Universe on the Pathway to SKA).

\section{References}

Becker, R. H., White, R. L., \& Helfand, D. J. 1995, ApJ, 450, 559

Condon, J. J., Cotton, W. D., Greisen, E. W., et al. 1998, AJ, 115, 1693

Metzger, B. D., Margalit, B., Kasen, D., \& Quataert, E. 2015, MNRAS, 454, 3311

Owen, F. N., Rudnick, L., Eilek, J., et al. 2014, ApJ, 794, 24

Perley, R. A., Chandler, C. J., Butler, B. J., \& Wrobel, J. M. 2011, ApJ, 739, L1

Tanvir, N. R., Levan, A. J., Fruchter, A. S., et al. 2013, Nature, 500, 547 